\title{
Vitamina D: una hormona poderosa
}

\author{
Vitamin D: a powerful hormone
}

La vitamina $D$ representa uno de los principales factores determinantes para el desarrollo de la vida en la tierra y para la evolución humana. Aproximadamente el 10\% al 20\% de los requerimiento de vitamina $\mathrm{D}$ de un organismo humano se puede obtener a través de la dieta y aproximadamente el 90\% del total de la vitamina $D$ necesaria tiene que ser fotosintetizada en la piel a través de la acción del sol (luz ultravioleta B (UV-B)) [I].

El raquitismo por deficiencia de vitamina $D$ fue la enfermedad de los jóvenes entre 1800 y principios del siglo $\mathrm{XX}$, con proporciones epidémicas en el norte de Europa, Norteamérica y Asia del Norte [2]. Sir Edward Mellanby (1919) demostró que esta enfermedad era debida a una deficiencia nutricional y se podía mejorar con suplementos de aceite de hígado de bacalao. McCollum (1922) demostró que el factor terapéutico en este aceite era una nueva vitamina, la vitamina $D$ [3,4]. Por su parte, Huldshinsky y sus compañeros de trabajo (1919) también demostraron que el raquitismo podía ser tratado con la luz ultravioleta (UV). Así mismo, Steenbock (1923) demostró que la luz UV inducía la formación de vitamina $D$ en las porciones grasas de los alimentos y en la piel, y mediante la irradiación de alimentos, logró proporcionar los medios por los cuales el raquitismo podría dejar de ser un problema médico importante.

Todos estos trabajos permitieron que los químicos aislaran e identificaran la estructura de la vitamina D entre 1919 y 1924, lo que llevó finalmente a la síntesis química de grandes cantidades de ella $[3,4]$ y su disponibilidad para el tratamiento farmacológico. En la figura I se ilustran las causas y posibles consecuencias de la deficiencia de vitamina $D$.

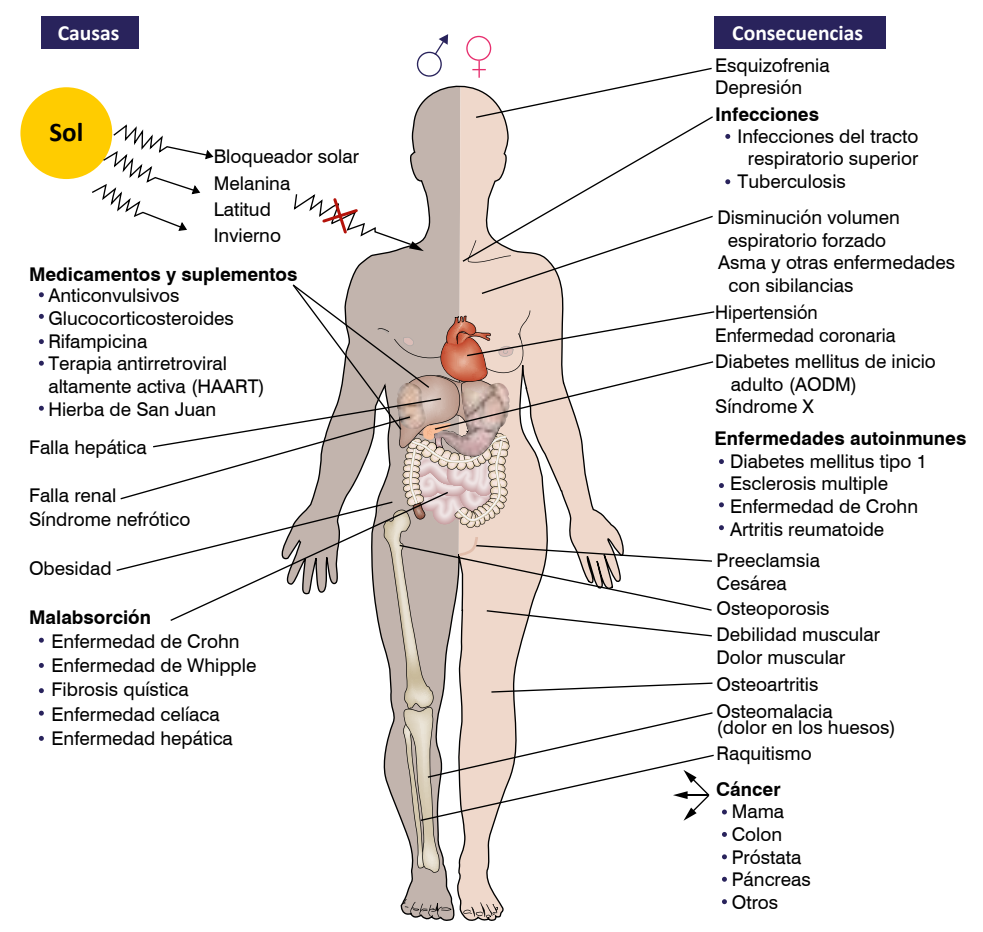

Figura I. Representación esquemática de las principales causas y posibles consecuencias de la deficiencia de vitamina $D$ sobre la salud humana. Tomado y modificado de "The vitamin D deficiency pandemic: A forgotten hormone important for health" por M.F. Holick, 2010, Public Health Rev, 32, p. 279. Copyright $\complement 2010$ por EHESP Presses. 
A principios de la década de 1960 se sabía poco sobre la función de la vitamina D y su efecto en la mineralización del esqueleto y, por lo tanto, en la prevención de las enfermedades como el raquitismo en los niños y la osteomalacia en los adultos. Con la aplicación de herramientas modernas de la bioquímica vino el descubrimiento de que la vitamina $D$ primero debe ser modificada por una 25-hidroxilación en el hígado seguida por una I $\alpha$-hidroxilación en el riñón para producir la hormona activa, la 1,25-dihidroxi vitamina $\mathrm{D}_{3}\left[\mathrm{I}, 25-(\mathrm{OH})_{2} \mathrm{D}_{3}\right]$. Este proceso es estrictamente regulado por retroalimentación y es uno de los principales sistemas endocrinos que controlan las concentraciones séricas de calcio y fósforo. Además, es uno de los principales reguladores de la masa y el bienestar óseo. Con la síntesis química de la I,25-(OH) $\mathrm{D}_{3}$ y muchos de sus análogos se ha brindado la posibilidad de tratar una serie de enfermedades metabólicas óseas como el raquitismo resistente a la vitamina D, el hipoparatiroidismo, la osteodistrofia renal y la osteoporosis [5].

Vale la pena resaltar que el sistema endocrino de la vitamina D (VDES; del inglés, Vitamin D Endocrine System), más allá de mantener la homeostasis del calcio y el fosfato, promueve la diferenciación e inhibe la proliferación de ciertas células, lo que sugiere un papel potencial en la quimioprevención del cáncer [6]. También se ha demostrado en varios modelos animales que la vitamina $D$ puede controlar ciertas enfermedades autoinmunes $[7,8]$ y proteger contra la aterosclerosis [9]. A pesar de todo el conocimiento actual, todavía existe un amplio camino por recorrer en el estudio y compresión del papel de la vitamina $D$ en la vida humana y constituye un importante campo de investigación actual.

En este número de Medicina \& Laboratorio, en el módulo "La Clínica y el Laboratorio", se incluye una revisión sobre la fisiología ósea y del balance fosfocálcico, titulada "Metabolismo mineral y óseo: visión general y sus métodos de medición", en la que se amplía el papel que desempeña la vitamina $D$ en la salud esquelética.

\section{lina Restrepo Givaldo}

Lina M. Restrepo Giraldo. MD, cMSc.

Clínica Medellín y Laboratorio Clínico Hematológico.

Medellín, Colombia, diciembre 2015

\section{Bibliografía}

1. Reichrath J, Zouboulis CC, Vogt T, Holick MF. Targeting the vitamin $D$ endocrine system (VDES) for the management of inflammatory and malignant skin diseases: An historical view and outlook. Rev Endocr Metab Disord 2016: 1-13.

2. Hess AF. The History of rickets. En: Hess AF, ed. Rickets, Including Osteomalacia and Tetany. Filadelfia, Estados Unidos: Lea \& Febiger; 1929: 22-37.

3. DeLuca HF. The transformation of a vitamin into a hormone: the vitamin D story. Harvey Lect 1979; 75: 333-379.

4. DeLuca HF, Schnoes HK. Vitamin D: recent advances. Annu Rev Biochem 1983; 52: 411-439.
5. Deluca HF. The vitamin D story: a collaborative effort of basic science and clinical medicine. FASEB J 1988; 2: 224-236.

6. Deeb KK, Trump DL, Johnson CS. Vitamin D signaIling pathways in cancer: potential for anticancer therapeutics. Nat Rev Cancer 2007; 7: 684-700.

7. Plum LA, Deluca HF. Vitamin D, disease and therapeutic opportunities. Nat Rev Drug Discov 2010; 9: 941-955.

8. DeLuca HF. Overview of general physiologic features and functions of vitamin D. Am J Clin Nutr 2004; 80: 1689S-1696S.

9. Reid IR, Bolland MJ. Role of vitamin D deficiency in cardiovascular disease. Heart 2012; 98: 609614. 